\title{
Interval training induces clinically meaningful effects in daily activity levels in COPD
}

\begin{abstract}
To the Editor:
Mounting evidence suggests that daily activity levels (DAL) in patients with chronic obstructive pulmonary disease (COPD) are markedly low compared with healthy age-matched individuals and are associated with poorer health status and prognosis [1]. COPD severity negatively impacts on DAL since patients with low DAL experience greater ventilatory, central haemodynamic and peripheral muscle oxygenation constraints during activities of daily living when compared with more physically active counterparts $[2,3]$. Although exercise training as part of pulmonary rehabilitation has shown to mitigate the aforementioned physiological constraints [4], there is no evidence of clinically meaningful improvements in DAL following pulmonary rehabilitation [5] as manifested by a mean increase of at least 1000 steps.day ${ }^{-1}$ [6]. This has been attributed to methodological shortfalls, such as lack of adequately controlled studies, small sample size, short duration of pulmonary rehabilitation programmes, application of activity monitors non-validated for COPD patients [5] and insufficient exercise intensities to induce true physiological training effects. Interval exercise training has been shown to allow application of intense loads to peripheral muscles that induce substantial physiological effects manifested by mitigation of respiratory and central haemodynamic limitations and partial restoration of peripheral muscle dysfunction in patients with diverse COPD severity $[7,8]$. In this context, it is reasoned that application of this training modality would allow transfer of the aforementioned physiological benefits into clinically meaningful improvements in DAL [2, 3]. Accordingly, the purpose of this randomised controlled study was to investigate the effect of a 12-week high-intensity interval exercise training programme in DAL in addition to usual care in patients with COPD.
\end{abstract}

The study was approved by the University Hospital Ethics Committee (Protocol ID-18367) and registered at the Clinical Trials online database (NCT02437994). 150 consecutive COPD patients were screened and referred for an outpatient 12-week pulmonary rehabilitation programme between September 2011 and September 2015. Baseline clinical assessment was part of the clinical care of COPD patients referred to the pulmonary rehabilitation service. DAL was assessed for seven consecutive days prior to the start, immediately following the completion of pulmonary rehabilitation and 12 weeks after the completion of pulmonary rehabilitation using a validated for COPD patients activity monitor (Actigraph GT3X, Actigraph LLC Pensacola, FL, USA) [9]. Patients were randomised in a 2:1 ratio into the interval training group (ITG) or the usual care group (UCG) using sealed opaque envelopes [10] in random block sizes of three and six. Data analysis was performed as per protocol. Exclusion criteria for participation in the pulmonary rehabilitation programme have been described elsewhere [8]. Medical treatment was not altered throughout the study in either of the groups. Patients with a minimum of four valid days (including weekends), counting only days with at least $480 \mathrm{~min}$ of wearing time during waking hours (as defined between 07:00 h-20:00 h) were contained in the analysis [11]. Vector magnitude units (VMU), the sum of movements in three planes over each minute, were used to quantify the intensity of DAL. Activity data were analysed using the ActiLife software (version 5.10.0). The interval programme included supervised exercise training consisting of cycling exercise 3 days.week ${ }^{-1}$ on electromagnetically braked cycle ergometers $[7,8]$. Patients assigned to ITG, exercised at a mean intensity of $130 \pm 18 \%$ of baseline peak work rate for $45 \mathrm{~min}$ by alternating 30 -s exercise intervals with 30 -s rest periods. The pulmonary rehabilitation programme included resistance training for upper and lower limbs with an intensity of 70\% of one repetition maximum, breathing retraining, dietary advice and education programme [4]. No specific education sessions on changes in DAL were included. The UCG group served as a control group not undertaking any of the components of the rehabilitation programme. To minimise the amount of bias experienced, health professionals who were blinded to group allocation performed all categories of assessment. Data are reported as mean \pm SD. Normal distribution was assessed using the Shapiro-Wilk test revealing that all data were normally distributed. Baseline characteristics between the two groups were compared using independent paired t-test. One-way ANOVA with repeated measures was employed to detect significant differences in DAL across different time points in the ITG. Two-way ANOVA with repeated measures was employed to evaluate intervention $\times$ time interaction effects. Pair-wise differences were identified using Tukey's honestly significant difference post hoc procedure.

Baseline characteristics were not different between the two groups (table 1). Significantly intervention $\times$ time interaction effects were observed between the ITG and the UCG for aerobic and functional capacities, 
respiratory symptoms, health-related quality of life, emotional status and all physical activity outcomes (table 1). ITG patients significantly and clinical meaningfully increased the mean number of steps per day (by $1094 \pm 1158$ steps $\cdot$ day $^{-1}$ or $27 \%$ from baseline; table 1). Furthermore, 38 (45\%) out of 85 patients exhibited an increase of $>1000$ steps.day ${ }^{-1}$ whilst six more patients (7\%) demonstrated an increase ranging between 900-1000 steps $\cdot$ day $^{-1}$. Time spent in sedentary intensity of activities significantly decreased whilst time spent in light, lifestyle, and moderate activities significantly increased following the pulmonary rehabilitation programme (table 1). In addition, ITG patients had an increase over UCG of $1475 \pm 1358$ steps $\cdot$ day $^{-1}$ or $36 \%$ from baseline following the pulmonary rehabilitation programme. Compared with baseline 12 weeks following completion of pulmonary rehabilitation, steps per day ( $4774 \pm 2692$ versus $4043 \pm 2484$ steps.day $^{-1}$ ) and time spent in light activities (159 \pm 65 versus $135 \pm 62 \mathrm{~min}$ ) remained significantly greater, whilst time spent in sedentary activities $(543 \pm 99$ versus $576 \pm 87 \mathrm{~min}$ ) was significantly lower in the ITG group.

TABLE 1 Outcomes before and after 12 weeks of high-intensity interval exercise training or usual care

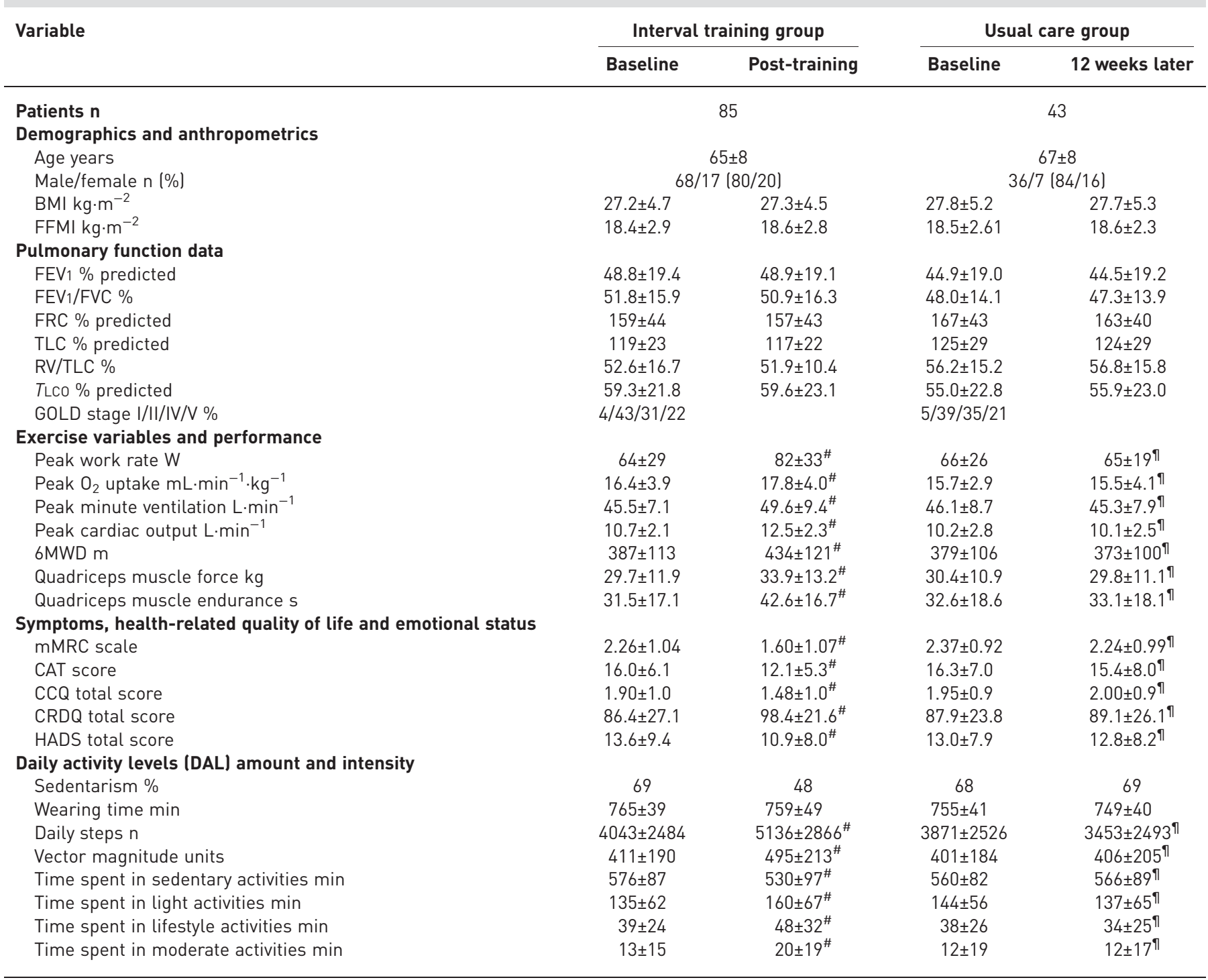

Data are presented as mean \pm SD, unless otherwise stated. Lung function was performed following American Thoracic Society (ATS)/European Respiratory Society (ERS) recommendations. Cardiac output assessed by impedance cardiography [8]. 6-min walk distance (6MWD) was measured as the best of two 6-min walk tests following ATS/ERS guidelines. Sedentarism was defined as a step count $<5000$ steps-day ${ }^{-1}$ [12]. Isometric quadriceps muscle force and endurance measured with the patient fixed in $90^{\circ}$ hip and knee flexion [13]. BMI: body mass index, FFMI: Fat free mass index. FEV1: forced expiratory volume in $1 \mathrm{~s}$; FVC: forced vital capacity; FRC: functional residual capacity; RV: residual volume; TLC: total lung capacity; TLCO: diffusing capacity of the lung for carbon monoxide; GOLD: Global Initiative for Obstructive Lung Disease; 6MWD: 6 min walk distance; mMRC: Modified Medical Research Council questionnaire; CAT: COPD Assessment Test; CCQ: Clinical COPD Questionnaire; CRDQ: Chronic Respiratory Disease Questionnaire; HADS: Hospital Anxiety Depression Scale. \#: within-group significant differences; ף: between-groups significant differences (interval training group versus usual care group). A p-value of $<0.05$ was considered significant. 
The present data demonstrate that in patients with COPD high-intensity interval exercise training as part of a comprehensive pulmonary rehabilitation programme can induce clinically meaningful improvements in DAL in terms of amount and intensity that persist for at least 12 more weeks post-rehabilitation. In addition, the importance of these novel findings is reinforced by the fact that the study was conducted in accordance with the recently published article for COPD patients' standardised procedure for DAL analysis [11] as well as by the inclusion of a control group [5].

In the present study, the observed clinically meaningful increase in daily number of steps observed in the ITG group following completion of pulmonary rehabilitation was accompanied by significant improvements in ventilatory, central haemodynamic and peripheral muscle capacities. In view of the fact that the intensity of the exercise training stimulus is a critical determinant of the magnitude of physiological adaptations that occur in response to training [14], high-intensity interval training portraits as more favourable for COPD patients who are unable to sustain moderate intensities for long periods of time to achieve substantial physiological effects due to symptom limitations of dyspnoea and leg discomfort [4]. Therefore, it is concluded that induction of true physiological adaptations following high-intensity interval training can be converted to clinically meaningful improvements in DAL in patients with COPD.

The content of the pulmonary rehabilitation programme in the present study did not include physical activity counselling interventions. However, it is anticipated that the decrease in respiratory symptoms, the improvement in health-related quality of life, anxiety, depression and emotional status that was observed following the pulmonary rehabilitation programme, might have positively impacted on behavioural status and thus have further contributed to achieve meaningful improvements in DAL. However, future studies aiming at investigating the association between physiological and health related outcomes with behavioural status for physical activity are warranted in order to support the abovementioned arguments.

Conventional pulmonary rehabilitation programmes have not in general shown significant increases in time spent at least in moderate activities in patients with COPD [5]. In this context, a recent study showed that training modalities mimicking everyday-life activities has proven to positively impact in DAL [15]. Interestingly, the authors found that following 12 weeks of Nordic walking training, walking intensity was significantly improved [15]. In our study, we found that time spent in sedentary activities decreased and time spent in lifestyle and moderate activities increased by $9 \pm 19$ and $7 \pm 11$ min.day ${ }^{-1}$, respectively. In addition, we found that the time spent in light activities remained significantly greater compared with baseline and 12 weeks following completion of pulmonary rehabilitation. Indeed, in COPD patients, the metabolic response during interval training seems comparable to the metabolic load during simple, self-paced domestic activities of daily life [16]. Thus, based on previous and these findings, it can be concluded that interval training can increase the intensity of everyday-life activities.

To the extent of our current knowledge, this is the first randomised control study demonstrating that high-intensity interval rehabilitative exercise training induces a clinically meaningful increase in DAL in patients with COPD.

0 @ERSpublications

12 weeks of interval training induces clinically meaningful effects in amount and intensity of daily activities in COPD http://ow.ly/rZXI3002awp

Zafeiris Louvaris ${ }^{1}$, Stavroula Spetsioti ${ }^{1}$, Eleni A. Kortianou ${ }^{2}$, Maroula Vasilopoulou ${ }^{1}$, Ioannis Nasis ${ }^{1}$, Georgios Kaltsakas ${ }^{3}$, Nikolaos G. Koulouris ${ }^{3}$ and Ioannis Vogiatzis ${ }^{1,3}$

${ }^{1}$ Faculty of Physical Education and Sports Sciences, National and Kapodistrian University of Athens, Athens, Greece. ${ }^{2}$ Dept of Physiotherapy, Technological Educational Institute of Central Greece, Lamia, Greece. ${ }^{3} 1$ st Dept of Respiratory Medicine, Pulmonary Rehabilitation Unit, National and Kapodistrian University of Athens, Sotiria Hospital, Athens, Greece.

Correspondence: Ioannis Vogiatzis, University of Athens, 1st Department of Critical Care Medicine, 3 Ploutarhou Str, 10675, Athens, Greece. E-mail: gianvog@phed.uoa.gr

Received: Feb 072016 | Accepted after revision: April 142016 | First published online: June 232016

Clinical trial: This study is registered at clinicaltrials.gov with identifier number NCT02437994.

Support statement: This work was partly funded by Innovative Medicines Initiative Joint Undertaking (IMU-JU \# 115011) through the Thorax Foundation as part of the PROactive project. Funding information for this manuscript has been deposited with FundRef.

Conflict of interest: None declared. 


\title{
References
}

1 Watz H, Pitta F, Rochester CL, et al. An official European Respiratory Society statement on physical activity in COPD. Eur Respir J 2014; 44: 1521-1537.

2 Kortianou EA, Aliverti A, Louvaris Z, et al. Limitation in tidal volume expansion partially determines the intensity of physical activity in COPD. J Appl Physiol (1985) 2015; 118: 107-114.

3 Louvaris Z, Kortianou EA, Spetsioti S, et al. Intensity of daily physical activity is associated with central hemodynamic and leg muscle oxygen availability in COPD. J Appl Physiol (1985) 2013; 115: 794-802.

4 Spruit MA, Singh SJ, Garvey C, et al. An official American Thoracic Society/European Respiratory Society statement: key concepts and advances in pulmonary rehabilitation. Am J Respir Crit Care Med 2013; 188: e13-e64.

5 Spruit MA, Pitta F, McAuley E, et al. Pulmonary rehabilitation and physical activity in patients with chronic obstructive pulmonary disease. Am J Respir Crit Care Med 2015; 192: 924-933.

6 Durheim MT, Smith PJ, Babyak MA, et al. Six-minute walk distance and accelerometry predict outcomes in chronic obstructive pulmonary disease independent of Global Initiative for Chronic Obstructive Lung Disease 2011 Group. Ann Am Thorac Soc 2015; 12: 349-356.

7 Vogiatzis I, Terzis G, Stratakos G, et al. Effect of pulmonary rehabilitation on peripheral muscle fiber remodeling in patients with COPD in GOLD stages II to IV. Chest 2011; 140: 744-752.

8 Nasis I, Kortianou E, Vasilopoulou M, et al. Hemodynamic effects of high intensity interval training in COPD patients exhibiting exercise-induced dynamic hyperinflation. Respir Physiol Neurobiol 2015; 217: 8-16.

9 Rabinovich RA, Louvaris Z, Raste Y, et al. Validity of physical activity monitors during daily life in patients with COPD. Eur Respir J 2013; 42: 1205-1215.

10 Doig GS, Simpson F. Randomization and allocation concealment: a practical guide for researchers. J Crit Care 2005; 20: 187-191.

11 Demeyer H, Burtin C, van Remoortel H, et al. Standardizing the analysis of physical activity in patients with COPD following a pulmonary rehabilitation programme. Chest $2014 ; 146: 318-327$.

12 Tudor-Locke C, Craig CL, Thyfault JP, et al. A step-defined sedentary lifestyle index: <5000 steps/day. Appl Physiol Nutr Metab 2013; 38: 100-114.

13 Allaire J, Maltais F, Doyon JF, et al. Peripheral muscle endurance and the oxidative profile of the quadriceps in patients with COPD. Thorax 2004; 59: 673-678.

14 Casaburi R, Patessio A, Ioli F, et al. Reductions in exercise lactic acidosis and ventilation as a result of exercise training in patients with obstructive lung disease. Am Rev Respir Dis 1991; 143: 9-18.

15 Breyer MK, Breyer-Kohansal R, Funk GC, et al. Nordic walking improves daily physical activities in COPD: a randomised controlled trial. Respir Res 2010; 11: 112.

16 Vaes AW, Wouters EF, Franssen FM, et al. Task related oxygen uptake during domestic activities of daily life in patients with COPD and healthy elderly subjects. Chest 2011; 140: 970-979.

\section{Silent aspiration in patients with exacerbation of COPD}

\author{
To the Editor:
}

Patients with chronic obstructive pulmonary disease (COPD) are susceptible to aspiration, probably due to discoordination between breathing and swallowing, cricopharyngeal muscle dysfunction, and changes in lung volume [1, 2]. TERADA et al. [3] found a significantly higher prevalence of an abnormal swallowing reflex in patients with COPD than in healthy controls. SHAKER et al. [4] found that patients with acute exacerbation of COPD (AECOPD) have a higher risk of aspiration. In this cross-sectional study, using the technetium-99m-sulfur colloid $\left({ }^{99 \mathrm{~m}} \mathrm{Tc}-\mathrm{SC}\right)$ salivary scintigraphy method that is believed to be more sensitive than techniques using in previous studies, the prevalence and risk factors of silent aspiration in hospitalised AECOPD patients was studied.

In this study, hospitalised AECOPD patients were recruited from the Guangzhou Institute of Respiratory Disease, First Affiliated Hospital of Guangzhou Medical University, Guangzhou, China, between December 2011 and August 2012. The study protocol was approved by the Scientific Research Ethics Review Committee of the First Affiliated Hospital of Guangzhou Medical University (approval number 2012/44). The clinical trial registration number is ChiCTR-CCH-12002918 (www.chictr.org.cn). Written informed consent was received from all patients participating in the study.

The inclusion criteria for patients with COPD were: age $\geqslant 50$ years; smoking history of $\geqslant 30$ years; medical history, physical examination, chest imaging and previous pulmonary function tests supportive of a diagnosis of COPD [5]; ratio of post-bronchodilator forced expiratory volume in $1 \mathrm{~s}(\mathrm{FEV} 1)$ to forced vital capacity $<70 \%$; and admission into hospital due to AECOPD [6]. The exclusion criteria were as follows: 